\title{
Passive mode locking by carbon nanotubes in a femtosecond laser written waveguide laser
}

Giuseppe Della Valle, Roberto Osellame, Gianluca Galzerano, Nicola Chiodo, Giulio Cerullo, Paolo Laporta, Orazio Svelto, Uwe Morgner, A. G. Rozhin, V. Scardaci, and A. C. Ferrari

Citation: Appl. Phys. Lett. 89, 231115 (2006); doi: 10.1063/1.2403912

View online: https://doi.org/10.1063/1.2403912

View Table of Contents: http://aip.scitation.org/toc/apl/89/23

Published by the American Institute of Physics

\section{Articles you may be interested in}

$320 \mathrm{fs}$ pulse generation from an ultrafast laser inscribed waveguide laser mode-locked by a nanotube saturable absorber

Applied Physics Letters 97, 111114 (2010); 10.1063/1.3486177

Graphene mode locked, wavelength-tunable, dissipative soliton fiber laser

Applied Physics Letters 96, 111112 (2010); 10.1063/1.3367743

Mechanical exfoliation of graphene for the passive mode-locking of fiber lasers

Applied Physics Letters 99, 121107 (2011); 10.1063/1.3641419

Ultra-short pulse generation by a topological insulator based saturable absorber

Applied Physics Letters 101, 211106 (2012); 10.1063/1.4767919

Graphene Q-switched, tunable fiber laser

Applied Physics Letters 98, 073106 (2011); 10.1063/1.3552684

Graphene mode-lockers for fiber lasers functioned with evanescent field interaction

Applied Physics Letters 96, 051122 (2010); 10.1063/1.3309669

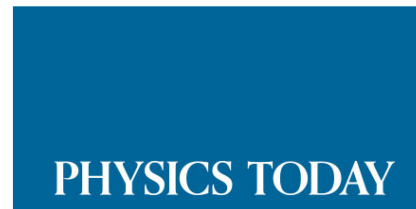

MANAGER'S GUIDE

WHITEPAPERS

\section{READ NOW}

Accelerate R\&D with

Multiphysics Simulation
PRESENTED BY

$\checkmark \subset \bigcirc M S \bigcirc L$ 


\title{
Passive mode locking by carbon nanotubes in a femtosecond laser written waveguide laser
}

\author{
Giuseppe Della Valle, ${ }^{\text {a) }}$ Roberto Osellame, Gianluca Galzerano, Nicola Chiodo, \\ Giulio Cerullo, Paolo Laporta, and Orazio Svelto \\ Istituto di Fotonica e Nanotecnologie-CNR, ULTRAS-INFM-CNR, Dipartimento di Fisica, Politecnico di \\ Milano, Piazza Leonardo da Vinci, 32, 20133 Milan, Italy \\ Uwe Morgner \\ Institut für Quantenoptik, University of Hannover, Welfengarten 1, Hannover D-30167, Germany
}

\author{
A. G. Rozhin, V. Scardaci, and A. C. Ferrari \\ Engineering Department, Cambridge University, 9 JJ Thompson Avenue, Cambridge CB3 OFA, \\ United Kingdom
}

(Received 11 July 2006; accepted 29 October 2006; published online 7 December 2006)

\begin{abstract}
The authors report on the first demonstration of mode locking in an active waveguide laser manufactured by femtosecond laser writing. The active waveguide is fabricated in an Er-Yb-doped phosphate glass, and the mode locker is a fiber-pigtailed saturable absorber device based on single-wall carbon nanotubes specially designed to efficiently operate at $1.5 \mu \mathrm{m}$. Transform-limited 1.6 ps pulses were observed in a ring laser cavity configuration. () 2006 American Institute of Physics. [DOI: 10.1063/1.2403912]
\end{abstract}

Recently, a great effort has been devoted to passive mode locking of waveguide lasers, because of their inherent simplicity and compactness with respect to fiber lasers. ${ }^{1,2}$ Er-Yb-doped glass waveguides can, in fact, generate large gains $(2-4 \mathrm{~dB} / \mathrm{cm})$ over short lengths, thus allowing compact cavities and high repetition rate operation without the need of harmonic mode locking with its associated instabilities. Such lasers will provide low-noise and inexpensive light sources for applications in optical communications, optically sampled analog-to-digital converters, ${ }^{3}$ and in spectral lineby-line pulse shaping for arbitrary optical wave form synthesis. ${ }^{4}$

An innovative technology for fabrication of optical waveguides is the direct writing by femtosecond laser pulses. ${ }^{5}$ This is a simple and low cost technique that avoids any photolithographic process and allows three-dimensional fabrication. In the last few years, the quality of femtosecond laser written waveguides has shown a substantial improvement, culminating in the demonstration of a $\mathrm{cw}$ waveguide laser. ${ }^{6}$ The next challenge consists in short pulse generation and passive mode locking using saturable absorbers appears to be the most promising solution. ${ }^{7}$

Classically, semiconductor saturable absorber mirrors are employed for passive mode locking of lasers. ${ }^{8}$ Recently, a new technology based on carbon nanotubes (CNTs) has emerged as an alternative for the realization of saturable absorbers. CNTs show strong saturable absorption with ultrafast recovery, ${ }^{9}$ polarization insensitivity, and high optical damage threshold; ${ }^{10}$ in addition, the possibility to tune their band gap in the near IR spectral range by selecting the appropriate diameter distribution makes them attractive for applications as photonic switches. ${ }^{11}$ Finally, they can be easily and cheaply assembled into polymer composites, allowing their integration into optical fiber communication systems. These characteristics make them ideally suited as saturable

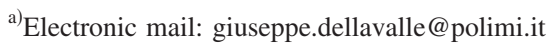

absorbers. ${ }^{12}$ In this letter we report on the first demonstration of a mode-locked laser based on a femtosecond laser written waveguide. The laser operates at $1.5 \mu \mathrm{m}$ and passive mode locking is obtained by means of a specially designed CNT saturable absorber, providing 1.6 ps transform-limited pulses at $16.7 \mathrm{MHz}$ repetition rate.

Direct waveguide writing is accomplished by a diodepumped cavity-dumped femtosecond $\mathrm{Yb}: \mathrm{KYW}$ oscillator, operating at $1040 \mathrm{~nm}$ wavelength, ${ }^{13}$ with $885 \mathrm{kHz}$ repetition rate, $250 \mathrm{~nJ}$ pulse energy, and $350 \mathrm{fs}$ pulse duration. The substrate is a commercial phosphate glass (QX from Kigre, Inc.) doped with 2 wt $\%$ of $\mathrm{Er}_{2} \mathrm{O}_{3}$ and 4 wt \% of $\mathrm{Yb}_{2} \mathrm{O}_{3}$. A very high numerical aperture objective $(100 \times$ oil immersion, 1.4 numerical aperture) is used to focus the femtosecond pulses $170 \mu \mathrm{m}$ inside the glass. A transverse writing configuration, i.e., with the sample translated in a direction perpendicular to the laser beam, is implemented with motorized stages at a translation speed of $50 \mu \mathrm{m} / \mathrm{s}$. Both end facets of the waveguides are polished after laser inscription. The writing parameters are optimized to obtain the best waveguide performance in terms of insertion losses.

The 36-mm-long waveguide employed in this work shows insertion losses of $1.9 \mathrm{~dB}$ when coupled to standard single mode fibers. The losses were measured at $1600 \mathrm{~nm}$ wavelength, outside the erbium absorption band. From nearfield measurements of waveguide and fiber modes, the coupling losses are estimated to be $\sim 0.25 \mathrm{~dB} /$ facet, and, consequently, propagation losses are evaluated to be of the order of $0.4 \mathrm{~dB} / \mathrm{cm}$. Previous gain measurements performed at $1.5 \mu \mathrm{m}$ (Ref. 14) showed net gain in the whole $C$ band, with $7.3 \mathrm{~dB}$ peak value at $1535 \mathrm{~nm}$ for an incident pump power of $460 \mathrm{~mW}$.

Figure 1 shows a schematic of the mode-locked waveguide laser in a ring cavity configuration. Two $976 \mathrm{~nm}$ laser diodes, providing $480 \mathrm{~mW}$ total incident power, are coupled to the waveguide in a bipropagating pumping scheme by means of wavelength division multiplexers (WDMs). A broadband fiber coupler is used to couple $5 \%$ of the intrac- 


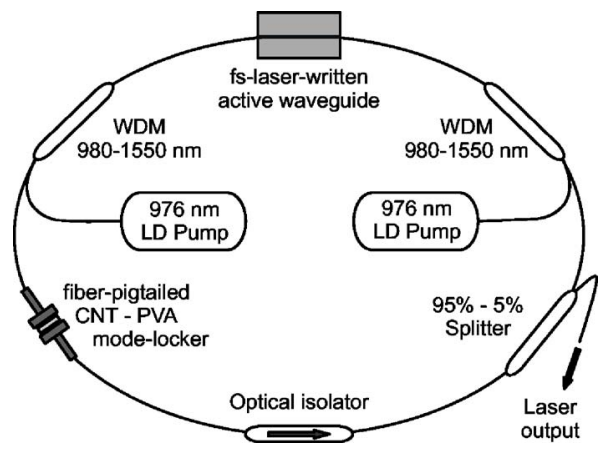

FIG. 1. Schematic setup of the ring laser cavity configuration.

avity radiation out of the ring resonator. Unidirectionality of the ring is imposed by an optical isolator, which also prevents mode-locking instabilities caused by back reflections. An index matching fluid is applied between the waveguide and fiber end facets, thus preventing Fabry-Pérot filtering effects, which could reduce the oscillating bandwidth. The mode locker is a single-wall CNT-spolymer film saturable absorber, packaged by sandwiching the film in the fiberpigtailed FC/PC connector with index matching fluid at both fiber ends (see Fig. 1). The CNTs are produced by laser ablation. ${ }^{15}$ High-power ultrasonication (Bioruptor, Diagenode) is utilized to disperse purified CNTs in water by using sodium dodecylbenzene sulphonate surfactant. The residual CNT bundles are removed by microfiltration. The resulting solution is then mixed with polyvinyl alcohol (PVA) and dried at room temperature to obtain a freestanding film with a thickness of $\sim 50 \mu \mathrm{m}$. The absorbance spectrum of the film is shown in Fig. 2: it has a broadband centered at $\sim 1.5 \mu \mathrm{m}$, with peak absorbance of about 0.36 (corresponding to $1.52 \mathrm{~dB}$ absorption, of which $0.6 \mathrm{~dB}$ is saturable). Such film has high laser damage threshold (more than $600 \mathrm{MW} / \mathrm{cm}^{2}$ ) and a saturation intensity of about $80 \mathrm{MW} / \mathrm{cm}^{2}$. A recovery time lower than $1 \mathrm{ps}$ is reported for CNT-polymide composites. ${ }^{9}$

Figure 3(a) shows the laser output spectrum recorded with an optical spectrum analyzer $(0.1 \mathrm{~nm}$ resolution bandwidth). Continuous wave laser action starts at $450 \mathrm{~mW}$ incident pump power. Self-starting single-pulse stable mode locking is observed just above laser threshold. No self$Q$-switching instabilities are observed and the mode-locking regime is stable for several hours. The laser output power is measured to be $\sim 0.1 \mathrm{~mW}$. Such a low value is due to the relatively high insertion losses along the ring cavity. In addition to the CNT-PVA film absorption, the packaging of the

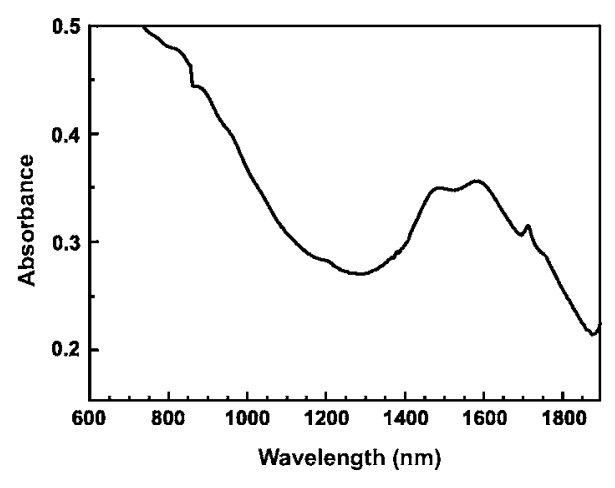

FIG. 2. Optical absorbance of the CNT-PVA film.
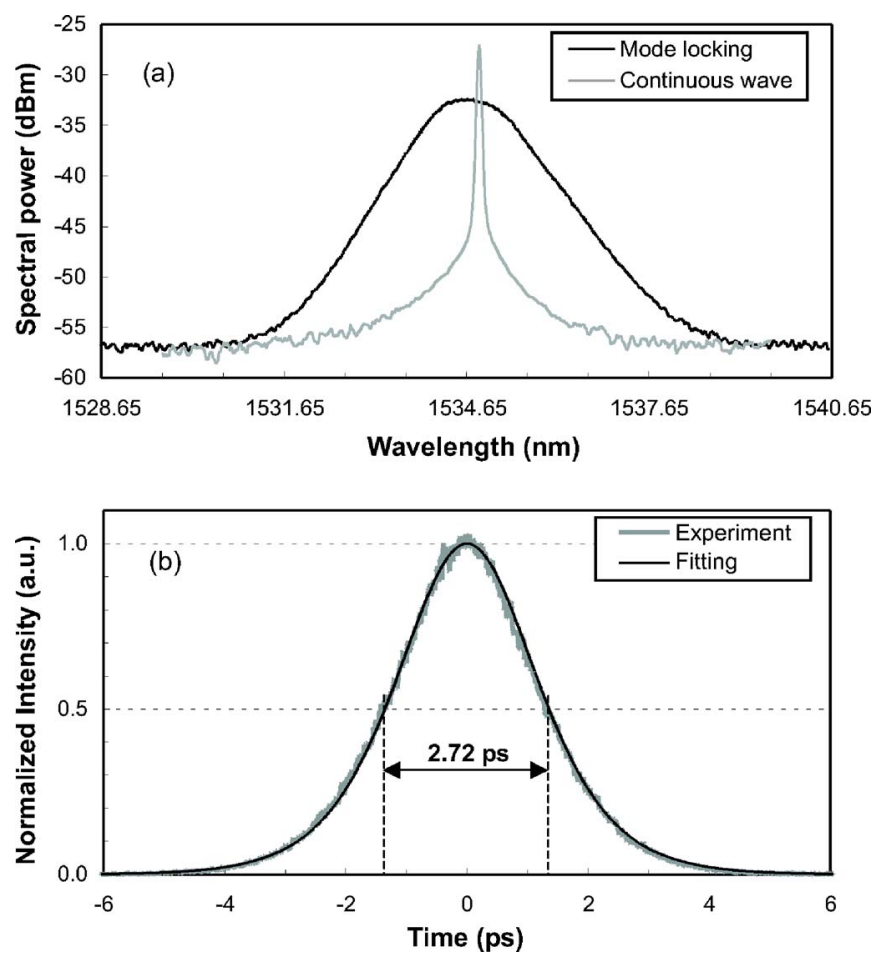

FIG. 3. (a) Laser output spectrum in continuous wave and mode-locking regimes. (b) Autocorrelation trace of the mode-locking pulse.

fiber-pigtailed mode locker is responsible for $2.8 \mathrm{~dB}$ extra losses, bringing the measured nonsaturated insertion loss to about $4.3 \mathrm{~dB}$. Taking into account WDMs and optical isolator insertion losses $(1.7 \mathrm{~dB})$, and cavity output coupling $(0.22 \mathrm{~dB})$, the overall ring cavity losses amount to $6.2 \mathrm{~dB}$, rather close to the net gain obtained in the waveguide.

The laser central wavelength in the mode-locking regime is $1535 \mathrm{~nm}$, and the full width at half maximum (FWHM) is $1.6 \mathrm{~nm}$, corresponding to $\Delta \nu=204 \mathrm{GHz}$. The pulse shape was investigated by a noncollinear autocorrelator, and the pulse autocorrelation trace is reported in Fig. 3(b). The experimental trace is well fitted considering a sech ${ }^{2}$ pulse as expected from saturable absorber passive mode-locking theory. ${ }^{7}$ The autocorrelation trace FWHM was measured to be $2.72 \mathrm{ps}$, corresponding to a pulse duration $\Delta \tau=1.76 \mathrm{ps}$. Taking into account the dispersion introduced by the $6 \mathrm{~m}$ standard single mode fiber connecting the laser to the autocorrelator $\left(16 \mathrm{ps} \mathrm{nm}^{-1} \mathrm{~km}^{-1}\right)$, the pulse duration at the output of the laser is $\Delta \tau_{p}=1.60 \mathrm{ps}$, resulting in a time bandwidth product $\Delta \tau_{p} \Delta \nu=0.329$, in fairly good agreement with the 0.315 value for transform-limited $\operatorname{sech}^{2}$ pulses. The pulse duration is in good agreement with that calculated from soliton mode-locking theory considering that the overall intracavity dispersion amounts to $-0.12 \mathrm{ps}^{2} .7$ The repetition rate of the current cavity is $16.74 \mathrm{MHz}$. Note that a much higher repetition rate can be obtained using a linear cavity configuration where the saturable absorber is directly placed on the waveguide facet. As an example, according to the saturation intensity of the nanotube absorber, $1 \mathrm{GHz}$ operation could be feasible with an intracavity average power of about $100 \mathrm{~mW}$. Such a power level was already demonstrated in similar waveguide lasers operated in $\mathrm{cw}^{6}$

To investigate the stability of the pulse train, we measured the radio-frequency spectrum of the pulses around the eighth harmonic of the repetition rate $(133.89 \mathrm{MHz})$ by 


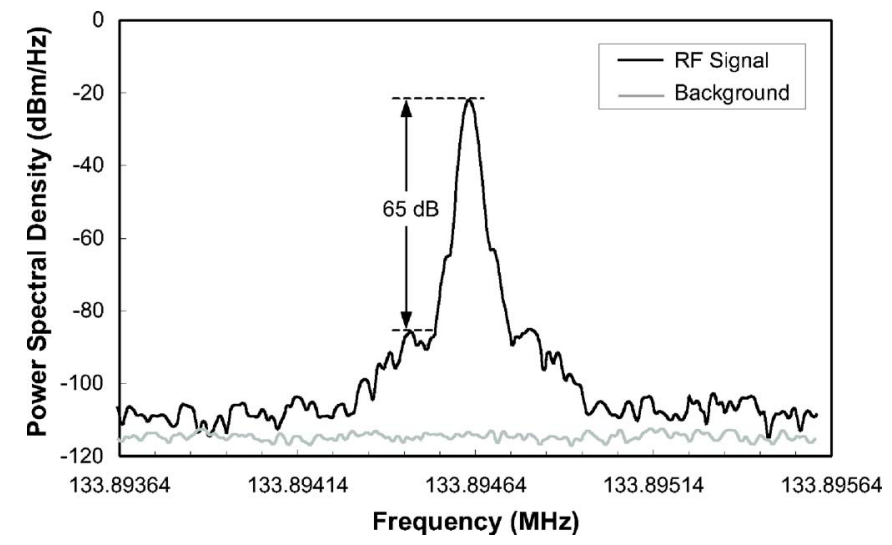

FIG. 4. rf spectrum of the mode-locking pulses measured at around the eighth harmonic of the repetition rate. Resolution bandwidth is $18 \mathrm{~Hz}$; jitter measurement bandwidth is $700 \mathrm{~Hz}$.

means of a fast photodiode ( $250 \mathrm{MHz}$ bandwidth) connected to an electrical spectrum analyzer. Figure 4 shows the signal spectrum together with the noise background due to photodetector thermal noise. A carrier-to-noise ratio of $65 \mathrm{~dB}$ indicates a low relative timing jitter $\Delta t / T$ (with $T$ being the train period); following the method reported in Ref. 16 we estimated $\Delta t / T \simeq 3 \times 10^{-4}$, corresponding to a jitter $\Delta t=19$ ps.

In conclusion, we demonstrated passive mode locking of a waveguide laser manufactured by direct femtosecond laser writing using a specially designed fiber-pigtailed CNT-PVA saturable absorber. Our work expands the capability of femtosecond laser writing towards manufacturing of complex photonic devices. An upgraded version of this technology, whereby a CNT absorber is incorporated into a monolithic laser structure, could provide very compact mode-locked laser sources with repetition rates in the $1-3 \mathrm{GHz}$ range. Moreover, by reducing intracavity losses and optimizing dispersion, it should be possible to exploit the whole Er gain bandwidth and achieve femtosecond operation.

The authors wish to thank G. Lanzani for useful discussion. Three of the authors (A.G.R., V.S., and A.C.F.) ac- knowledge funding from EPSRC Grant No. GR/S97613/01 and from Advance Nanotech Inc. A.C.F. acknowledges funding from The Royal Society and from The Leverhulme Trust. U.M. acknowledges support from the European Community Access to research infrastructure action, contract R113-CT2003-506350 (Centre for Ultrafast Science and Biomedical Optics).

${ }^{1}$ E. R. Thoen, E. M. Koontz, D. J. Jones, D. Barbier, F. X. Kaertner, E. P. Ippen, and L. A. Kolodziejski, IEEE Photonics Technol. Lett. 12, 149 (2000).

${ }^{2}$ J. B. Schlager, B. E. Callicoatt, R. P. Mirin, N. A. Sanford, D. J. Jones, and J. Ye, Opt. Lett. 28, 2411 (2003).

${ }^{3}$ P. W. Juodawlkis, J. C. Twichell, G. E. Betts, J. J. Hargreaves, R. D. Younger, J. L. Wassermann, F. J. O’Donnell, K. J. Ray, and R. C. Williamson, IEEE Trans. Microwave Theory Tech. 49, 1840 (2001).

${ }^{4}$ Z. Jiang, D. S. Seo, D. E. Leaird, and A. M. Weiner, Opt. Lett. 30, 1557 (2005).

${ }^{5}$ K. M. Davis, K. Miura, N. Sugimoto, and K. Hirao, Opt. Lett. 21, 1729 (1996).

${ }^{6}$ R. Osellame, N. Chiodo, G. Della Valle, G. Cerullo, R. Ramponi, P. Laporta, A. Killi, U. Morgner, and O. Svelto, IEEE J. Sel. Top. Quantum Electron. 12, 277 (2006).

${ }^{7}$ F. X. Kaertner, J. Aus der Au, and U. Keller, IEEE J. Sel. Top. Quantum Electron. 4, 159 (1998).

${ }^{8}$ U. Keller, Nature (London) 424, 831 (2003).

${ }^{9}$ Y.-C. Chen, N. R. Raravikar, L. S. Shadler, P. M. Ajayan, Y.-P. Zhao, T.-M. Lu, G.-C. Wang, and X.-C. Zhang, Appl. Phys. Lett. 81, 975 (2002).

${ }^{10}$ A. G. Rozhin, Y. Sakakibara, H. Kaktaura, S. Matsuzaki, K. Ishida, Y. Achiba, and M. Tokumoto, Chem. Phys. Lett. 405, 288 (2005).

${ }^{11}$ H. Kataura, Y. Kumazawa, Y. Maniwa, I. Umezu, S. Suzuki, Y. Ohtsuka, and Y. Achiba, Synth. Met. 103, 2555 (1999).

${ }^{12}$ T. R. Schibli, K. Minoshima, H. Kataura, E. Itoga, N. Minami, S. Kazaoui, K. Miyashita, M. Tokumoto, and Y. Sakakibara, Opt. Express 13, 8025 (2005).

${ }^{13}$ R. Osellame, N. Chiodo, G. Della Valle, S. Taccheo, R. Ramponi, G. Cerullo, A. Killi, U. Morgner, M. Lederer, and D. Kopf, Opt. Lett. 29, 1900 (2004)

${ }^{14}$ G. Della Valle, R. Osellame, N. Chiodo, S. Taccheo, G. Cerullo, P. Laporta, A. Killi, U. Morgner, M. Lederer, and D. Kopf, Opt. Express 13, 5976 (2005).

${ }^{15}$ S. Lebedkin, P. Schweiss, B. Renker, S. Malik, F. Hennrich, M. Neumaier, C. Stoermer, and M. M. Kappes, Carbon 40, 417 (2000).

${ }^{16}$ D. von der Linde, Appl. Phys. B: Photophys. Laser Chem. 39, 201 (1986). 